\title{
Demographic and clinical characteristics of gastric cancer patients in north of Iran, Mazandaran province, 2008-2014
}

\author{
Seyed Khalil Mousavi ${ }^{1}$, Ghasem Janbabai ${ }^{2}$, Bizhan Kouchaki ${ }^{1}$, Hanieh Borhani ${ }^{3}$, Masoumeh \\ Rashidi ${ }^{3}$, Ebrahim Salehifar* ${ }^{4 *}$ \\ ${ }^{1}$ Students Research Committee, Faculty of Pharmacy, Mazandaran University of Medical Sciences, Mazandaran, Sari. Iran \\ ${ }^{2}$ Department of Hematology and Oncology, Cancer Research Center, Imam Khomeini Hospital, Mazandaran University of Medical \\ Sciences, Sari, Iran \\ ${ }^{3}$ Cancer Research Center, Imam Khomeini Hospital, Mazandaran University of Medical Sciences, Sari, Iran \\ ${ }^{4}$ Department of Clinical Pharmacy, Pharmaceutical Sciences Research Center, Mazandaran University of Medical Sciences, Sari, Iran
}

\begin{abstract}
Gastric cancer has been known as a poor prognosis disease with a high degree of mortality. In Iran, it has been increased during the previous two decades, especially in northern part of the country. The aim of this study was to determine the demographic and clinical characteristics of gastric cancer in Mazandaran province. All patients with gastric cancer diagnosis treated in the Imam Khomeini educational hospital or Touba Polyclinic, a university affiliated center, from March 2008 to March 2014 were included. Demographic data, the symptoms at the time of presentation, tumor size, type of tumor, lymph node involvement, status in terms of metastatic disease, therapeutic plan and type of chemotherapy regimens were gathered from patients' medical records. Statistical analysis was performed using SPSS, version 16. Totally, 643 patients were investigated. The mean age of patients was 64.34 years and men were more affected than women. "Time to relapse" and "time to progression" were 13 and 6.5 months, respectively. The most common initial symptoms at diagnosis were nausea, vomiting and epigastric pain (47.4\%). Only in $6.3 \%$, the disease was diagnosed at the initial stages. The most common site of involvement was cardia (almost 40\%) and antrum (32\%). More than 91\% of tumors were adenocarcinoma. DCF (Docetaxel, Cisplatin, 5FU) (43.3\%), Xeloda (15.2\%), DOX (Docetaxel, Oxaliplatin) (13.8\%) and ECF (Epirubicin, Cisplatin.5FU) $(9.2 \%)$ were the most common used regimens. There was a trend for improved survival with DCF regimen $(\mathrm{P}=0.08)$. Most gastric cancer patients were referred in the advanced stage or metastatic disease. The demographic characteristics are consistent with other previous reports. DCF regimen may have some advantages over other chemotherapy regimens.
\end{abstract}

Keywords: Gastric cancer, demographic, chemotherapy, DCF, Mazandaran

Pharm Biomed Res 2015; 1(1): 32-36

DOI: 10.18869/acadpub.pbr.1.1.32

\section{Introduction}

Gastric cancer has been known as a poor prognosis disease with a high degree of mortality. It is one of the most common cancers with a diverse geographic variability worldwide. Latin America, Eastern Asia, parts of Europe and Middle East are among regions with a high incidence of gastric cancer, while the lowest rates are in parts of Africa and North America. Patients are often diagnosed in the advanced stages (1). Seventy percent of gastric cancer occurs in the developing countries, with a higher incidence in men compared to women. In Iran, it is the most common cancer in men and the second frequent cancer in women after breast cancer. Worldwide, it was the leading cause of mortality of cancer deaths until three decades ago.

As other part of the world, the high incidence and poor prognosis of gastric cancer is still a great health system concern in the country. Considering the cancer mortality in both sexes, gastric cancer is the most common cancerrelated mortality in Iran $(2,3,4)$. Although the incidence and mortality of gastric cancer has been declined in many countries including United States, the absolute number of new cases 
per year is increasing, mainly due to aging of the world population. In our country, there was an increase in the incidence of gastric cancer especially at the Northern provinces such as Mazandaran (5).

Treatment of cancer usually is accompanied with different modalities such as surgery, radiation therapy and chemotherapy. In Gastric cancer, the type of treatment should be selected based on the stage of the disease, clinical status of the patients, his or her comorbidities and preference (6). Worldwide, gastrectomy is the most widely used approach for treatment of gastric cancer. Chemotherapy has been widely used in treatment of different stage of gastric cancer. Over eighty percent of patients who die of gastric cancer experience a local recurrence of the disease. This is the basis for adjuvant chemotherapy. Neoadjuvant chemoradiotherapy or preoperative chemoradiotherapy may also be selected for gastric cancer, based on the location of the tumor. The decision which chemotherapy regimen is a better choice for a given patient is based on the stage of disease and the patients' baseline characteristics. Commonly, regimens based on the 5-fluorouracil (5-FU), Oxaliplatin, Cisplatin, Docetaxel and Paclitaxel are used as monotherapy or combination chemotherapy $(7,8)$. To update the demographic and clinical characteristics of gastric cancer patients, this study has been conducted in Mazandaran province of Iran.

\section{Materials and methods}

This study was a descriptive observational study. All patients with gastric cancer diagnosis who were been treated in the Emam Khomeini educational hospital or Touba polyclinic, a university affiliated center, from March 2008 until March 2014 were included. The study was approved by ethics committee of Mazandaran University of Medical Sciences. Different demographic and clinical characteristics including sex, age, weight, height, job, the date of first visit, patient's symptoms at the time of diagnosis, macro and microscopic data of the tumor including the anatomic location of involvement (cardia, antrum, fundus, etc), tumor size, type (adenocarcinoma or other type), tumor invasion to lymph nodes or distant metastasis, the plan of treatment selected including surgery, radiation therapy and or chemotherapy and type of chemotherapy gathered. "Time to relapse" and "time to progression" and survival were compared for different chemotherapy regimens. Statistical analysis has been done with SPSS version 16 . Descriptive statistics were used to express the results. Analysis of variance (ANOVA) was executed to compare different regimens in terms of "time to relapse", "time to progression" and survival rate. $\mathrm{P}<0.05$ was considered as a significant difference.

\section{Results}

Totally six hundred and forty three patients were included in the study. Demographic characteristics of patients have been presented in table 1.

Table 1 Demographic and clinical characteristics of patients

\begin{tabular}{|c|c|}
\hline Sex (male \%) & 68.3 \\
\hline Age $($ mean \pm SD $)$ & $64.35 \pm 14$ \\
\hline $\begin{array}{l}\text { Initial symptoms } \\
\text { nausea, vomiting and epigastric pain } \\
\text { fatigue, weakness and melena } \\
\text { weight loss } \\
\text { dysphagia }\end{array}$ & $\begin{array}{l}47.4 \% \\
34.2 \% \\
10.5 \\
2.6 \\
\end{array}$ \\
\hline $\begin{array}{l}\text { Stages } \\
1 \\
2 \\
3 \\
4 \\
\end{array}$ & $\begin{array}{l}27(6.3 \%) \\
57(13.3 \%) \\
103(24 \%) \\
243(56.5 \%) \\
\end{array}$ \\
\hline $\begin{array}{lllll}\text { frequency }(\%) & \text { of } & \text { surgery } & \text { in } \\
\text { different stages } & & & \\
\text { stage 1 } & & & \\
\text { stage 2 } & & & & \\
\text { stage 3 } & & & & \\
\text { stage 4 } & & & & \\
\end{array}$ & $\begin{array}{l}24(96 \%) \\
48(98 \%) \\
79(85.9 \%) \\
53(27.7 \%)\end{array}$ \\
\hline $\begin{array}{l}\text { Tumor location } \\
\text { Cardia } \\
\text { Antrum } \\
\text { Body } \\
\text { Fundus } \\
\text { Other } \\
\end{array}$ & $\begin{array}{l}134(39.6 \%) \\
109(32.2 \%) \\
36(10.7 \%) \\
11(3.3 \%) \\
48(14.2) \\
\end{array}$ \\
\hline $\begin{array}{l}\text { Type of tumor } \\
\text { Adenocarcinoma } \\
\text { other types }\end{array}$ & $\begin{array}{l}403(91.2 \%) \\
39(8.8 \%)\end{array}$ \\
\hline $\begin{array}{l}\text { Metastasis } \\
\text { Liver } \\
\text { Ascites and peritoneal involvement } \\
\text { paraaort } \\
\text { Lung } \\
\text { Liver and paraaort } \\
\text { Liver and Lung } \\
\text { Others }\end{array}$ & $\begin{array}{l}80(46.9 \%) \\
45(26.6 \%) \\
14(8.2 \%) \\
8(4.7 \%) \\
5(2.9 \%) \\
2(1.2 \%) \\
16(9.4 \%)\end{array}$ \\
\hline
\end{tabular}

The men were two times most affected than women. The mean age of patients was 64.35 years. Time to relapse and time to progression were 13 and 6.5 months, respectively. The most common symptoms at the time of diagnosis were nausea, vomiting and epigastric pain $(47.4 \%)$, followed by fatigue and weakness. 
Cardia (40\%) and antrum (32\%) were sites most involved in gastric cancer. The type of tumor was adenocarcinoma in more than $91 \%$ of cases. Whereas only $6.3 \%$ of patients were in stage 1 , the majority $(56.4 \%)$ were in stage 4 at the time of referring to the oncology clinic. Liver was the most common site of metastasis $(47 \%$ liver metastasis) and also there were cases that had simultaneously liver metastasis with peritoneum involvement, ascites and paraaortic metastasis. Totally, liver metastasis was occurred in almost $80 \%$ of gastric cancer patients. Sixty four percent of patients were undergone surgery before referring to oncologist. 191 patients were in stage 4, among them, $53(28 \%)$ were undergone surgery. Neoadjuvant therapy was done for $39(6 \%)$ of cases. Relapse was occurred in $10.1 \%$ of patients, whereas the liver was the most common site of relapse. Progression was documented in 28 medical records. Liver involvement and ascites were common features of progression. Time to relapse, Time to progression and overall survival were not statistically different between four common used regimens. Though, DCF was associated with a trend of improved overall survival $(\mathrm{P}=0.082)$ (Table 2).

Table 2 Outcome variables of different chemotherapy regimens

\begin{tabular}{l|c|c|c|c|c|c}
\hline Variables/Regimens & ECF* & DOX* & Xeloda* & DCF* & Total & P-value* \\
& & & & & $12.77 \pm 8.432$ & 0.643 \\
\hline Time to relapse & $10.6 \pm 2.302$ & $11.57 \pm 8.662$ & $10.4 \pm 7.701$ & $14.83 \pm 9.892$ & $5.91 \pm 2.836$ & 0.711 \\
\hline Time to progression & NA & $6.5 \pm 3.464$ & $4.67 \pm 1.155$ & $6.17 \pm 3.403$ & $3.69 \pm 2.094$ & 0.825 \\
\hline Number of courses & $4.04 \pm 1.255$ & $3.62 \pm 1.623$ & $3.71 \pm 3.189$ & $3.63 \pm 1.813$ & & \\
\hline
\end{tabular}

*DCF: Docetaxel.Cisplatin.5FU; DOX:Docetaxel.Oxaliplatin. Capecitabine; ECF: Epirubicin.Cisplatin.5FU; Xeloda: Capecitabine; P-value: ANOVA test between four chemotherapy regimens; NA: not available data

\section{Discussion}

This study which was designed to determine the demographic characteristics of gastric cancer in Mazandaran showed that the observed characteristics were in concordance with results of other studies.

In this study the number of male gastric cancer patients was 2.15 times more than females. This result is in agreement with the results of the study conducted by Biglarian and colleagues (9) (male: female ratio was 2.5 ) and is also similar to the results of the study performed by Shafigh and colleagues (10) (male:female ratio was (2.89). Mean age of the patients at the time of diagnosis was $65 y$ for males and $63.7 y$ for females. Mean age of the patients in Biglarian and colleagues (9) study was 59.39 for males and 56.22 for females. In three other studies conducted by Roshanayi and colleagues (11), Akhavan and colleagues (12) and Dehkordi and colleagues (13) mean age of the patients were 58.7, 59 and 46.2, respectively. These results are relatively similar to the results of the present study. As a general rule, age of the gastric cancer patients is an important prognostic factor. The more the age of the patient at the time of diagnosis is, the poorer prognosis of the patients will be (4). It seems that changes happened in the mean age of the population in our country have affected the prevalence of gastric cancer and its prognosis. This fact could be more important in areas of the country that the disease is more prevalent. In a study performed by Asmarian and colleagues (14) data gathered from 366 cities throughout the country between the 1382-1386 showed that gastric cancer is more prevalent in northern areas of the country especially in Mazandaran, Golestan, Ardabil and Kordestan provinces (14).

An important problem in gastric cancer is that most patients are diagnosed in late stages of the disease so it is difficult to treat them effectively and this delay in diagnosis significantly decreases survival time of the patients. In our study population only $6 \%$ of the patients were diagnosed at stage I and in $80 \%$ of metastatic cases, liver metastasis were present which in turn predispose the patient to a poor prognosis. 
Results of studies conducted in other area of the country show similarities in stage of the disease at the time of the diagnosis of the patients. Biglarian and colleagues (9) reported that in their study population only $26.8 \%$ of patients diagnosed at stage I of the disease and $37 \%$ of them diagnosed at stage IV. Keyhanian and colleagues (15) study showed that in Ramsar, $46.5 \%$ of patients were at stage IV and $18.8 \%$ were at stage III when diagnosed. $31.9 \%$ of the patients had metastasis and among them $78 \%$ had liver metastasis. In Davudabadi and colleagues study $60 \%$ of patients diagnosed at late stages (16). Similar results were found by Somi and colleagues in East Azarbayjan (17).

These results indicate that unfortunately and as a common problem, in majority of gastric cancer patients the disease is diagnosed at late stages. The reason for this is probably difficulty in making a differential diagnosis at early stages of the disease and lack of proper and cost-effective screening program for diagnosis of the patients. These factors seemingly contribute in high mortality of gastric cancer patients.

Studying chemotherapeutic regimen of our patients revealed that DCF regimen is prescribed more than ECF, DOX and Xeloda. Furthermore there was a trend toward longer "mean survival time" in patients receiving DCF regimen although mean "time to recurrence" and mean "time to progression" were not significantly different. These findings indicate relative superiority of DCF regimen to other chemotherapeutic regimens. However, because of high cost of this regimen (mainly because of high cost of docetaxel) and because this superiority is not an unchangeable fact for all patients it is not recommendable to use DCF for all gastric cancer patients. In other studies, adding docetaxel to $\mathrm{CF}$ regimen resulted in more favorable outcomes. For example in a phase 3 clinical trial performed by Cutsem and colleagues (18), adding docetaxel to $\mathrm{CF}$ regimen lead to improvement in quality of life, time to progression and survival time of the patients ( $\mathrm{p}<0.001)$. In an another study conducted in MD Anderson Cancer Center by Ajani(19) it has been shown that the rate of complete response, time to progression, overall survival and quality of life were significantly better in patients received DCF rather than $\mathrm{CF}$ regimen. In this study "time to progression" was 5.6 and 3.7 for DCF and CF groups, respectively. Mean survival time were 9.2 in DCF group versus 8.6 in $\mathrm{CF}$ group. These statistical differences do not necessarily mean a significant clinical superiority of DCF regimen. Especially when taking into account limitations in financial resources exists for health systems, it is not reasonable to consider high cost DCF regimen as the first choice in all gastric cancer patients.

Cutsem et al (18) also showed that in their study population the most common sites involved in gastric cancer were body (44\%), anthrum (25\%) and fundus (12\%), respectively. Keyhanian and colleagues (15) reported antrum (45.1\%) and cardia $(29.2 \%)$ as the most common sites of involvement in gastric cancer patients and $98.8 \%$ of tumors were diagnosed as adenocarcinomas.

In Sumi and colleagues(17) study population, $41.1 \%$ of tumors were at proximal third, $25.3 \%$ were at medial third and $25.3 \%$ were at distal third of the stomach (17). In this study $96 \%$ of tumors were of adenocarcinoma type. Unlike the above mentioned studies, cardia was the most common site of involvement in our study, followed by antrum. Similar to other reports, adenocarcinoma type was very common in our gastric cancer patients. An important factor which affects the prognosis of the patients and response to treatment is the site of tumor. According to the previous studies tumors of the cardia show a poorer response to the treatment relative to tumors located at more distal parts of the stomach and mortality rate is also higher in cardia tumors (7). A comparison between the results of the present study with results of the studies performed in other areas of the country shows that unfortunately most gastric tumors develop at sites of the stomach that respond poorly to the treatment.

In the present study the most prevalent symptoms among the patients when first came to the clinic were nausea, vomiting, epigastric pain $(47.4 \%)$ and the most prevalent clinical presentations were weakness, tiredness, melena $(34.2 \%)$ and weight loss $(10.5 \%)$. So our results were similar to the results of other studies. Sumi and colleagues (17) reported abdominal pain (26.7\%), dysphagia( $30 \%$ ) and loss of appetite $(28 \%)$ as the most prevalent symptom of their patients. In Sharifi and colleagues (20) study, $85.6 \%$ of the gastric cancer patients reported epigastric pain as their first symptom.

\section{Conclusion}

Most aspects of the demographic characteristics are consistent with other studies. Unlike some previous reports, cardia was the most common site on involvement on our study. Most gastric cancer patients referred in the advanced stage or metastatic disease. DCF regimens was prescribed more than ECF, DOX and Xeloda. 
This regimen may have some advantage over the other chemotherapy regimens. There was a trend toward longer "mean survival time" in patients receiving DCF regimen although mean "time to recurrence" and mean "time to progression" were not significantly different.

\section{Acknowledgment}

This study was supported by a grant from Research and Technology deputy of

\section{References}

1. Khushalani N. Cancer of the esophagus and stomach. Mayo Clinic Proc 2008; 83:712-22.

2. Movahedi M, Afsharfard A, Moradi A, Nasermoaddeli A,Khoshnevis J, Fattahia F, et al. Survival rate of gastric cancer in Iran. JRMS 2009; 14:367-73.

3. Yazdannbod A, Arshi S, Derakhshan MH, Sadjadi AR,Malekzadeh R. Gastric cardia cancer; the most common type of upper gastrointestinal cancer in Ardabil, Iran: An endoscopy clinic experience. Arch Iran Med 2001; 4:76-9.

4. Yazdani Charati J, Zare S. Ghorbanpour E. Shabankhani B. Demographic and Geographical Pattern of Mortality Rate from Stomach Cancer and Related Factors in Mazandaran Province From 2001 to 2005. J Mazand Univ Med Sci 2009; 20:2-7.

5. Naeeme SA, Naemeh S, Behzad M, Amir K. Mapping of Stomach Cancer Rate in Iran Using Area-to-Area Poisson Kriging. J Health Syst Res 2012;8:681-7

6. NCCN Clinical Practice Guidelines in Oncology: Gastric Cancer (Including the proximal $5 \mathrm{~cm}$ of the stomach). Version 1.2014. Available at http://bit.ly/lLKqEg. Accessed July 30, 2014.

7. Catalano V. Labianca R, Beretta G, Gatta G, Braud F, Van Cutsem E. Gastric cancer. Crit Rev Oncol Hemat 2009; 54:209-41.

8. Woll E. Chemotherapy in Gastric Cancer. Anti Cancer Res 2008; 28:1213-20.

9. Biglaryan A. Hajizade A. Gohari M. Khodabakhshi R. Survival Analysis of gastric cancer patients and its related factors. Kowsar Med J 2007; 12:345-55.

10. Shafigh A. Siadati S. Shafayi Sh. Epidemiological studies of cancer in hospitals of Babol University of Medical Sciences 13years. J Babol Univ Med Sci 2005;7:73-8 (Persian).

11. Roshanaei Gh. Kazemnejad A. Sadighi S. Assessment of the Survival Risk Factors in Patients with Gastric Cancer in Cancer Institute of Imam Khomeni Hospital between2003-2007. J Zanjan Univ Med Sci 2012; 20:40-50 (Persian).

12. Akhavan A, Bashardoust N, Moghimi M, Seifadini A, Salehi M. Survival rate of patients suffering fromgastric
Mazandaran University of Medical Sciences. We would like to appreciate all patients and their family who responded patiently to our questions.

\section{Conflict of interest}

The authors declared no potential conflict of interest with respect to the authorship, and/or publication of this study.

adenocarcinoma of Yazd city. Kowsar Med J 2011; 16:39-44.

13. Moghimi-Dehkordi B, Rajaei Fard A, Tabatabaei $H$, Zeighami B, Safaee A, Tabei SZ. Survival analysis using the Cox regression model in patients with gastric cancer. J Epidemiol 2007; 3:19-24.

14. Asmarian N, Kavousi A, Salehi M, Mahaki B. Mapping of Stomach Cancer incidence rate in Iran from 2003-2007 using Area-to-Area Poisson Kriging. Health Syst Res 2012:8:1-7.

15. Keyhanian Sh, Farhadifar N, Fotoukian Z, Pouya M, Saravi M. Epidemiologic and malignancy indices of gastric cancer in patients referred to oncology clinic at ramsar emam sajjad hospital during 2002-2009. J Shahid Sadoughi Univ Med Sci 2012; 20: 110-8 (Persian).

16. DavoodAbadi A, Sharifi H, Erfan N, Dianati M. An Epidemiologic and clinical survey on Gastric Cancer Patients Referd to Shahid Beheshti Hospital Of Kashan(1994-2001). The Razi J Med Sci 2003; 10:21122.

17. Somi M, Alizadeh N, Mirinezhad, SK, Jazeyeri E, Sadegi M, Farhang S. Diagnosis and treatment of gastric cancer patients and their problems in East Azerbaijan Province. Med J Tabriz Univ Med Sci 2010; 32:57-63 (Persian).

18. Cutsem E, Moiseyenko V, Tjulandin S, Majlis A, Constenla M, Boni C, Rodrigues A, Fodor M, Chao Y, Voznyi E, Risse M,an Ajani J. Phase III Study of Docetaxel and Cisplatin Plus Fluorouracil Compared With Cisplatin and Fluorouracil As First-Line Therapy for Advanced Gastric Cancer: A Report of the V325 Study Group. J Clin Oncol 2006; 24:4991-7.

19. Ajani A. The role of docetaxel in gastric cancer. EJC Supplements 2006; 4:4-9.

20. Sharifei H, Dianati M, Masoud A. A study on the frequency of anatomic site, clinical sign and laboratory findings of stomach cancer patients referring to kashan shahid beheshti hospital during 1993-1999. Armaghanedanesh 1999; 13-14:43-8 (Persian) 